\title{
Effects of treatment change in patients with neovascular age-related macular degeneration; Results from the Czech National Registry
}

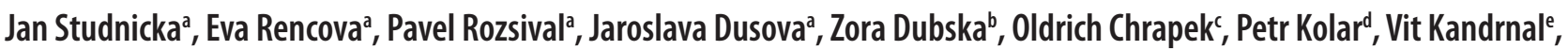 \\ Sarka Pitrova ${ }^{f}$, Jiri Rehak ${ }^{c}$
}

\begin{abstract}
Aims. To determine the effectiveness of second line treatments in patients with neovascular AMD who did not respond adequately to primary treatment.

Methods. Retrospective, multicentre assessment. The frequency of primary treatment failure and outcomes of subsequent secondary treatment were assessed according to the type of primary treatment, type of CNV and change in BCVA over a 12 month period.

Results. At the time of assessment 750 entries (750 treated eyes, 725 treated patients) had follow-up longer than 12 months. A treatment change required $7.7 \%$ subjects treated with ranibizumab, $20.5 \%$ with pegaptanib and $22 \%$ with PDT and verteporfin. Average BCVA of all patients at the beginning of primary treatment was $50.7 \pm 3$ letters and 43 \pm 3.5 letters in $12^{\text {th }}$ month $(P<0.001)$. The mean decrease in BCVA was $7.7 \pm 0.6$ letters during the first 6 months of observation. During the next 6 months, no significant change occurred. The change of primary therapy was required on average after $6.5 \pm 2.1$ months.

Conclusion. BCVA loss was the most significantly decelerated in patients who received ranibizumab as a secondary therapy following unsuccessful treatment with pegaptanib sodium.

Key words: age-related macular degeneration, choroidal neovascular membrane, visual acuity, ranibizumab, pegaptanib, photodynamic therapy with verteporfin

Received: June 18, 2012; Accepted: October 22, 2012; Available: November 28, 2012

http://dx.doi.org/10.5507/bp.2012.100

a Department of Ophthalmology, Faculty of Medicine in Hradec Kralove, Charles University in Prague and University Hospital in Hradec Kralove, Czech Republic

${ }^{b}$ Department of Ophthalmology, $1^{\text {st }}$ Faculty of Medicine, Charles University in Prague and General University Hospital in Prague

'Department of Ophthalmology, University Hospital Olomouc

${ }^{d}$ Department of Ophthalmology, University Hospital Brno

eInstitute of Biostatistics and Analyses, Faculty of Medicine and Faculty of Science, Masaryk University Brno

fPrivate Eye Clinic, Prague

Corresponding author: Petr Kolar, e-mail:pe-kolar@seznam.cz
\end{abstract}

\section{INTRODUCTION}

Age-related macular degeneration (AMD) is a disease characterized by degenerative changes of choroid mainly in the macular area. It is the most common cause of blindness in the population above 65 years in developed countries $^{1}$. The slowly progressive atrophic form of AMD affects the majority of patients. A rapidly progressive wet form, characterized by choroid neovascular membrane (CNV), develops in approximately $10 \%$ of cases ${ }^{2}$. Without treatment, the wet form of AMD leads to loss of 2.7 lines (13.5 letters) on the ETDRS chart in 1 year and 4 lines (20 letters) in 2 years $^{3}$.

Verteporfin (Visudyne ${ }^{\mathrm{TM}}$, Novartis Pharma AG) was approved in March 2000 by the FDA in the USA for treatment with photodynamic therapy (PDT) in patients with mostly classic CNV. The first anti-vascular endothelial growth factor (VEGF) drug approved for the treatment of the wet form of AMD was pegaptanib sodium (Macugen, Pfizer Inc.). This is a ribonucleic acid aptamer with high affinity for the VEFG $\mathrm{A}_{165}$ isomer. On binding with VEGF
$A_{165}$ prevents the ligation of VEGF molecules to endothelial cell VEGFR-2 receptors and stops their activation $^{4}$. Another drug approved for anti-VEGF treatment is ranibizumab (Lucentis, Novartis Pharma AG Europe, Genentech, Inc. USA), which is a fragment of the humanize monoclonal antibody against several isoforms of VEGF A (VEGF $A_{165}$, VEGF $A_{121}$ and VEGF $A_{110}$ ) (ref. ${ }^{5}$ ). Bevacizumab (Avastin, F. Hoffmann-La Roche Ltd, Genentech, Inc. USA) is a humanized monoclonal antibody to vascular endothelial growth factor A that is approved for the treatment of metastatic colorectal cancer. Although this drug is not approved for intraocular use in the Czech Republic, it has been used as an off - label intravitreal therapy in specific cases.

The treatment of patients with wet AMD is centralized to 9 tertiary referral centres in the Czech Republic (University Hospital Brno, University Hospital Hradec Kralove, University Hospital Kralovske Vinohrady Prague, University Hospital Olomouc, University Hospital Ostrava, University Hospital Plzen, Masaryk Hospital Usti nad Labem, Central Military Hospital Prague, General 
University Hospital Prague). Anonymised data on treatment efficacy and safety have been entered consecutively into the Czech national database AMADEUS (Age related MAcular DEgeneration in patientS in the Czech Republic) since September 2008. The main aim of the registry is to collect basic epidemiologic data on patients diagnosed with wet AMD in the Czech Republic, document standard diagnostic and therapeutic patterns and assess treatment efficacy in standard clinical practice. The data collection is independent of all treatment decisions, does not affect patient access to treatment and fully complies with all ethical as well as legal requirements for non-interventional data collection in the Czech Republic. All patients have given a written informed consent for primary and secondary treatment, as well as data collection.

The main aim of this analysis was to assess wet AMD secondary therapy treatment efficacy following failure of primary therapy.

\section{METHODS}

Our analysis included records from all 9 centres that cooperated on the national, multicentre registry Amadeus. The analysis criteria were as follows: enrolment between 06.10.2008 and 29.10.2010, minimum 12-month followup period, age more than 55 years, confirmed diagnosis of wet AMD, all types of CNV, treatment naïve patients, initial best corrected visual acuity score (BCVA) between 70 and 35 letters on ETDRS chart (equivalent of 20/40 - 20/200 on Snellen chart) if eye with worse BCVA was affected and $70-15$ letters on ETDRS chart (equivalent of 20/40 - 20/400 on Snellen chart) if the eye with better BCVA was affected.

All patients underwent a fluorescein angiography (FA) and OCT examination before treatment with PDT and/or anti-VEGF was initiated. FA was subsequently carried out only in case of therapeutic doubt. OCT examination was also performed prior to each pegaptanib, ranibizumab and PDT application. PDT with verteporfin was indicated in patients with predominantly classic $\mathrm{CNV}$, lesion size up to 4 Disc Areas (DA), pegaptanib and ranibizumab therapy was indicated in patients with all types of membranes, lesion size up to 8 DA (occult CNV's were treated only in cases of signs of activity in the retina such as bleeding, or hard exudates in the macula or worsening of BCVA by more than 1 line of the ETDRS chart within 3 months).

Records were excluded from analysis if another cause of CNV (myopia, angioid streaks, etc.) was confirmed, if the treated eye was infected,, if the FA revealed the lesion was bigger than $8 \mathrm{DA}$, predominantly fibrotic $\mathrm{CNV}$, and allergy to verteporfin, pegaptanib, ranibizumab and/or to fluorescein were present. The evaluation excluded patients with polypoidal choroidal vasculopathy (PCV) proven to FA before treatment. Indocyanine green angiography (ICGA) was not performed due to lack of availability of dye in the Czech Republic.

The analysis focused on treatment change due to primary therapy failure, defined as a decrease in BCVA by 15 or more letters on the ETDRS chart, and/or increase in intraretinal or subretinal fluid with an increase in CRT of more than 100 microns on OCT and/or an increase in CNV leakage confirmed by FA during the treatment. PDT with verteporfin was carried out according to the standard protocol; pegaptanib sodium was administered according to summary of product characteristic (SPC) at regular 6-week intervals. Subjects treated with ranibizumab initially received three injections in monthly intravitreal followed by "pro re nata" (PRN) application (i.e. the patients received further injections if the reapplication criteria were met).

During the 12-month period, at each visit all patients underwent examination of the BCVA on the ETDRS charts, anterior and posterior ocular segment examination, fundus photography, optical coherence tomography mainly focused on CRT and FA for identification of type and extent of CNV. The frequency of follow-up was minimally once per three months in the 9 tertiary referral centres. Patients treated with PDT with verteporfin and ranibizumab were reassessed every 3 months at the tertiary referral centres; patients treated with pegaptanib were assessed in those centres every 6 weeks. In addition, patients were seen monthly in outpatient ophthalmology clinics. In case of worsening of BCVA by more than 1 line on the ETDRS chart within 1-month period, patients were referred to the tertiary referral centre for reassessment. Treatment assessment was recorded in the $3^{\text {rd }}, 6^{\text {th }}$ and $12^{\text {th }}$ months. The primary objective of our analysis was to identify the primary treatment frequency failure and subsequent treatment patterns. The secondary objective was to assess BCVA change according to a different type of primary and secondary treatment and CNV type.

The statistical significance of differences between groups of patients was tested by Kruskal Wallis $\mathrm{H}$ test (for continuous parameters), Wilcoxon test (for continuous paired data), Mann Whitney $U$ test and maximum likelihood chi square test (for categorical data). Statistical significance was set at the 0.05 probability level. Analysis was conducted with SPSS 19.0.1. (IBM Corporation, 2010).

The primary treatment selection and choice of secondary treatment were fully within the competence of treating ophthalmologists at the tertiary referral centres. The reasons for treatment change in the case of unsatisfactory primary therapy were decrease in BCVA by 15 or more letters on the ETDRS chart, and/or increase in intraretinal or subretinal fluid with an increase in CRT by more than 100 microns on OCT and/or increase of CNV leakage confirmed by FA. The selection of drugs (verteporfin, pegaptanib sodium and ranibizumab) for secondary treatment was not driven by any specific protocol. Treating physicians worked in this respect independently and their decisions were based on the results of assessments, standard clinical practice of their centres and availability of treatment.

The study was conducted in accordance with the current version of the Declaration of Helsinki. 


\section{RESULTS}

From a total of 750 records ( 750 eyes, 725 patients) with a confirmed wet form of AMD, 454 eyes (60.5\%) were treated with ranibizumab, 146 eyes (19.5\%) with pegaptanib sodium and 150 eyes $(20 \%)$ with PDT with verteporfin. The treatment change was required in 35 eyes (7.7\%) treated initially with ranibizumab, 30 eyes $(20.5 \%)$ with pegaptanib sodium and 33 eyes $(22 \%)$ treated with PDT. Of a total of 96 patients who required a treatment change, 51 were women and 45 men. The mean age of the sub-cohort was $74.3 \pm 1.6$ years. Details regarding type of CNV confirmed by FA at the beginning of treatment are shown in Table 1.

The initial mean BCVA of the whole cohort was $50.7 \pm$ 3 letters on the ETDRS chart (median 52.5 letters). The mean BCVA in the $3^{\text {rd }}$ month was $50.4 \pm 2.8$ letters (median 52 letters) and $43 \pm 3.5$ (median 40.5 letters) in $6^{\text {th }}$ month and $43 \pm 3.5$ (median 40.5 letters) in the $12^{\text {th }}$ month $(P<0.001)$. The mean decrease in BCVA during the first 6 months of observation was $7.7 \pm 0.6$ letters. There was no significant change in BCVA between the $6^{\text {th }}$ and $12^{\text {th }}$ months. The mean BCVA at the beginning of treatment with PDT with verteporfin was $49.6 \pm 5$ letters (median 50 letters). BCVA at the end of 12 months observation period was $40.6 \pm 6.1$ letters (median 42 letters) $(P=0.002)$. The initial mean BCVA at in case of patients treated with ranibizumab was $52.9 \pm 4.7$ letters (median 55 letters) and $48.4 \pm 5.9$ letters (median 53 letters) in patients treated with pegaptanib sodium. BCVA following 12 months of treatment with ranibizumab and pegaptanib sodium was $47.6 \pm 5.5$ letters (median 45 letters) $(P=0.168)$ and $40.4 \pm 6.6$ letters (median 35.5) $(P=0.068)$ respectively. Details regarding the change of BCVA according to the primary therapy are summarized in Table 2. Table 3 shows the change in BCVA according to type of CNV. The mean duration of the primary therapy was $6.5 \pm 2.1$ months. The majority of patients in the PDT subgroup with verteporfin $(97 \%)$ were diagnosed with the predominantly classic CNV. Treatment change was

Table 1. Type and size of the CNV at the beginning of treatment.

\begin{tabular}{|c|c|c|c|c|c|c|}
\hline & & \multirow{2}{*}{ Total } & \multicolumn{3}{|c|}{ Primary therapy } & \multirow{2}{*}{$P^{1}$} \\
\hline & & & Visudyne & Lucentis & Macugene & \\
\hline \multicolumn{7}{|l|}{ Eyes $\mathrm{N}=98$} \\
\hline \multirow[t]{3}{*}{ CNV type N (\%) } & $\mathrm{PC}$ & $48(49.0 \%)$ & $31(93.8 \%)$ & $11(31.4 \%)$ & $6(20.0 \%)$ & \multirow{3}{*}{$<0.001$} \\
\hline & $\mathrm{OC}$ & $39(39.8 \%)$ & $1(3.1 \%)$ & $21(60.0 \%)$ & $17(56.7 \%)$ & \\
\hline & MC & $11(11.2 \%)$ & $1(3.1 \%)$ & $3(8.6 \%)$ & $7(23.3 \%)$ & \\
\hline \multirow[t]{2}{*}{$\mathrm{CNV}$ average size $\mathrm{N}(\%)$} & $<2 \mathrm{DD}$ & $43(43.9 \%)$ & $20(60.6 \%)$ & $10(28.6 \%)$ & $13(43.3 \%)$ & \multirow{2}{*}{0.147} \\
\hline & $\geq 2 \mathrm{DD}$ & $55(56.2 \%)$ & $13(39.4 \%)$ & $25(71.5 \%)$ & 17 (56.6\%) & \\
\hline
\end{tabular}

${ }^{1}$ Statistical significance of ML chi square test for categorical data and statistical significance of Kruskal Wallis $\mathrm{H}$ test for continuous data

Table 2. The best corrected visual acuity at the end of 12-month observation period.

\begin{tabular}{|c|c|c|c|c|c|c|c|}
\hline & & \multicolumn{5}{|c|}{ Primary therapy } & \multirow[b]{2}{*}{$P^{1}$} \\
\hline & & & $\begin{array}{c}\text { Visudyne } \\
\mathrm{N}=33 \\
\end{array}$ & & $\begin{array}{c}\text { Lucentis } \\
\mathrm{N}=35\end{array}$ & $\begin{array}{l}\text { Macugen } \\
\mathrm{N}=30\end{array}$ & \\
\hline \multirow{10}{*}{$\begin{array}{l}\text { BCVA } \\
\text { mean } \\
(5-95 \% \mathrm{CI}) \\
\text { median } \\
\left(5^{\text {th }}-95^{\text {th }} \text { percentile }\right)\end{array}$} & At entry & $\begin{array}{l}49.6 \\
50.0\end{array}$ & $\begin{array}{l}(44.7-54.6) \\
(29.0-77.0)\end{array}$ & $\begin{array}{l}52.9 \\
55.0\end{array}$ & $\begin{array}{l}(48.2-57.6) \\
(30.0-70.0)\end{array}$ & $\begin{array}{ll}48.4 & (42.4-54.3) \\
53.0 & (16.0-74.0)\end{array}$ & 0.995 \\
\hline & After 3 months & 49.4 & $(45.4-53.5)$ & 54.8 & $(49.9-59.7)$ & $46.4(40.8-51.9)$ & \multirow{2}{*}{0.223} \\
\hline & & 50.0 & $(28.0-65.0)$ & 55.0 & $(30.0-75.0)$ & $45.5(23.0-75.0)$ & \\
\hline & $P^{2}$ & & 0.899 & & 0.167 & 0.068 & \\
\hline & After 6 months & 45.3 & $(40.3-50.4)$ & 50.1 & $(45.1-55.1)$ & $42.1 \quad(37.1-47.2)$ & \multirow{2}{*}{0.382} \\
\hline & & 45.0 & $(15.0-68.0)$ & 50.0 & $(25.0-72.0)$ & $41.0(19.0-64.0)$ & \\
\hline & $P^{2}$ & & 0.086 & & 0.321 & 0.031 & \multirow{3}{*}{0.794} \\
\hline & After 12 months & 40.6 & $(34.5-46.7)$ & 47.6 & $(42.1-53.1)$ & $40.4(33.7-47.0)$ & \\
\hline & & 42.0 & $(3.0-68.0)$ & 45.0 & $(24.0-75.0)$ & $35.5(13.0-69.0)$ & \\
\hline & $P^{2}$ & & 0.002 & & 0.168 & 0.068 & \\
\hline
\end{tabular}

${ }^{1}$ Statistical significance of Mann Whitney U test

${ }^{2}$ Statistical significance of Wilcoxon test for paired samples, tested for entry and time after 3, 6 and 12 months 
Table 3. Development of the BCVA according to type of CNV.

\begin{tabular}{|c|c|c|c|c|c|c|}
\hline & & \multicolumn{5}{|c|}{ CNV type } \\
\hline & & $\begin{array}{c}\mathrm{PC} \\
\mathrm{N}=48\end{array}$ & & $\begin{array}{c}\mathrm{OC} \\
\mathrm{N}=39\end{array}$ & $\begin{array}{c}\mathrm{MC} \\
\mathrm{N}=11\end{array}$ & $P^{1}$ \\
\hline \multirow{11}{*}{$\begin{array}{l}\text { BCVA } \\
\text { mean } \\
(5-95 \% \\
\text { CI }) \\
\text { median } \\
\left(5^{\text {th }}-95^{\text {th }}\right. \\
\text { percentile })\end{array}$} & \multirow{2}{*}{ At entry } & $51.0(47.0-55.0)$ & 51.7 & $(47.0-56.4)$ & $43.2(32.3-54.0)$ & \multirow{2}{*}{0.170} \\
\hline & & $51.5(30.0-75.0)$ & 55.0 & $(24.0-75.0)$ & $42.0(15.0-74.0)$ & \\
\hline & \multirow{2}{*}{ After 3 months } & $50.6(46.8-54.5)$ & 52.0 & $(47.2-56.8)$ & $43.7(35.7-51.7)$ & \multirow{2}{*}{0.129} \\
\hline & & $50.0(30.0-70.0)$ & 54.0 & $(23.0-75.0)$ & $40.0(26.0-70.0)$ & \\
\hline & $P^{2}$ & 0.977 & & 0.936 & 0.755 & \\
\hline & \multirow{2}{*}{ After 6 months } & $44.3(40.1-48.6)$ & 48.7 & $(43.9-53.5)$ & $44.3(36.5-52.0)$ & \multirow{2}{*}{0.869} \\
\hline & & $44.0(19.0-70.0)$ & 50.0 & $(22.0-72.0)$ & $40.0(28.0-64.0)$ & \\
\hline & $P^{2}$ & 0.002 & & 0.236 & 0.929 & \\
\hline & \multirow{2}{*}{ After 12 months } & $41.3(36.6-46.1)$ & 44.8 & $(38.5-51.0)$ & $44.3(35.6-52.9)$ & \multirow{2}{*}{0.711} \\
\hline & & $40.5(14.0-69.0)$ & 49.0 & $(12.0-74.0)$ & $40.0(22.0-65.0)$ & \\
\hline & $P^{2}$ & $<0.001$ & & 0.068 & 0.878 & \\
\hline
\end{tabular}

${ }^{1}$ Statistical significance of Mann Whitney U test.

${ }^{2}$ Statistical significance of Wilcoxon test for paired samples, tested for entry and time after 3, 6 and 12 months.

Table 4. Primary and secondary therapy.

\begin{tabular}{lccc}
\hline Primary therapy & Visudyne $(\mathrm{N}=33)$ & Lucentis $(\mathrm{N}=35)$ & Macugen $(\mathrm{N}=30)$ \\
\hline Number of doses & \multicolumn{3}{c}{} \\
\hline Mean & 1.2 & 3.1 & 3.5 \\
$5-95 \%$ CI & $1.0 ; 1.3$ & $2.7 ; 3.5$ & $2.7 ; 4.4$ \\
Median & 1.0 & 3.0 & 4.0 \\
$10^{\text {th }}-90^{\text {th }}$ percentile & $1.0 ; 2.0$ & $2.0 ; 5.0$ & $1.0 ; 7.0$ \\
\hline Successive therapy & \multicolumn{3}{c}{} \\
\hline Lucentis & $27(81.8 \%)$ & - & $29(96.7 \%)$ \\
Macugen & $6(18.2 \%)$ & $25(71.4 \%)$ & - \\
Visudyne & - & $10(28.6 \%)$ & $1(3.3 \%)$ \\
\hline Time of change in treatment $($ in months $)$ & & \\
\hline Mean & 4.8 & 7.9 & 5.9 \\
$5-95 \%$ CI & $3.3 ; 6.3$ & $6.7 ; 9.0$ & $7.6 ; 7.2$ \\
Median & 3.0 & 8.0 & 7.0 \\
$10^{\text {th }}-90^{\text {th }}$ percentile & $2.0 ; 10.0$ & $3.0 ; 12.0$ & $1.0 ; 10.0$ \\
\hline
\end{tabular}

required, mainly due to insufficient treatment effect, in 33 cases (22\%); average duration of treatment was 4.8 months. The average number of laser treatments in this subgroup was 1.2. Subsequent treatment was performed in 27 eyes $(81.8 \%)$ with ranibizumab and 6 (18.2\%) eyes with pegaptanib sodium.

In the subgroup of 146 eyes primarily treated with pegaptanib sodium, a treatment change was required for 30 eyes $(20.5 \%)$ following 5.9 months of treatment and 3.5 applications, mainly due to insufficient treatment response. Subsequent treatment was performed in 29 eyes (96.7 \%) with ranibizumab and in 1 eye (3.3\%) with PDT with verteporfin.
In the subgroup primarily treated with ranibizumab $(n=454)$ the treatment change was required for 35 eyes (7.7\%) after 7.9 months; patient received on average 3.1 applications of the ranibizumab, mainly due to insufficient treatment. Subsequent treatment was performed in 25 eyes $(71.4 \%)$ with pegaptanig sodium and in 10 eyes (28.6\%) with PDT with verteporfin (Table 4 ).

\section{DISCUSSION}

All patients were treated at tertiary ophthalmology centres and the treatment reflected standard clinical prac- 
tice. Our analysis focused on patients with the wet form of AMD treated with ranibizumab, pegaptanib sodium and PDT with verteporfin.

The TAP (Treatment of Age - related Macular Degeneration with Photodynamic Therapy) study reported a loss of $\geq 15$ letters on the ETDRS charts in $39 \%$ of treated eyes that received on average 3.5 laser treatments during the 12 -month period ${ }^{6}$. In our group primarily treated with PDT we found a slightly smaller percentage of eyes that had been changed due to inefficiency of primary therapy with PDT with verteporfin. The average number of laser treatment in our group was smaller than in the TAP study. We tend to assume that the lower number of PDT applications in our subgroup was due to the availability of other treatment options and an early treatment change.

Ranibizumab was applied on a PRN basis which was also used in the PrONTO (Prospective OCT Imaging of Patients with Neovascular AMD Treated with Intraocular Ranibizumab) and SUSTAIN (Safety and Efficacy of Flexible Dosing Regimen of Ranibizumab in Neovascular Age Related Macular Degeneration) studies. Compared with an average of 5.6 injections of ranibizumab in the PRONTO study and 5.7 injections in the SUSTAIN study was found in our observation the lower average number of ranibizumab injections ${ }^{7,8}$. We believe that the low number of applications was influenced by longer follow-up periods in the maintenance phase and limited budget for the treatment of wet AMD in the Czech Republic.

The VEGF Inhibition Study in Ocular Neovascularization (VISION) reported that the intravitreal application of $0.3 \mathrm{mg}$ of pegaptanib sodium in 6-week intervals during a one-year treatment resulted in a loss of less than 15 letters on the ETDRS chart in $70 \%$ of patients. In $6 \%$ of patients BCVA improved by at least 3 lines on ETDRS chart $^{9}$. Quiram et al. documented $\geq 15$ letters decrease in BCVA on the ETDRS chart in $10 \%$ of patients with newly diagnosed CNV. The mean follow-up period was 9.1 months and the mean number of injections per patient was 4.7 (ref. $^{10}$ ). Our group of patients with documented poor response to the primary treatment with pegaptanib sodium was greater than that of Quiram et al. and smaller than that in the VISION trial. The average number of injections corresponds to the results of Quiram et al.

In the subgroup treated with PDT with verteporfin there was no significant difference in the BCVA decrease during the first and second half of the observation period. In other words, the disease progressed irrespective of the secondary treatment type applied. The same trend was observed in the subgroup treated initially with ranibizumab and subsequently with selective anti VEGF $\mathrm{A}_{165}$ blocker pegaptanib sodium. If the primary therapy failed during the first six months of the observation period, the disease progression usually continued even if the patients received a secondary therapy.

Disease progression was significantly slowed down in eyes unsuccessfully treated with pegaptanib sodium if they were subsequently treated with ranibizumab. The additional effect of ranibizumab on CNV not responding to pegaptanib sodium can be explained by its effect on other isoforms of VEGF A, mainly isoforms VEGF $\mathrm{A}_{121}$ and VEGF $A_{110}$ (ref. $^{5}$ ).

When we compared the BCVA according to the type of $\mathrm{CNV}$, we found a sharp reduction in visual acuity in eyes affected by predominantly classic CNV. When we compared development of the BCVA in all three groups with the natural development of the neovascular form of AMD (ref. ${ }^{3}$ ), we found that the disease progression was significantly decelerated in all treated groups of patients, despite the need for treatment change and limited effect of PDT and pegaptanib sodium as a subsequent treatment.

Data from our databases can provide additional information to the information from interventional clinical trials, can increase our understanding of the treatment management of patients with the wet form of AMD in standard clinical practice, and help us to optimize treatment patterns in cases of ineffective treatment.

\section{CONFLICT OF INTEREST STATEMENT}

Author's conflict of interest disclosure: The authors stated that there are no conflicts of interest regarding the publication of this article.

\section{ACKNOWLEDGEMENT}

Grant from Novartis Pharma AG was received for the national registry AMADEUS.

Participating AMADEUS Clinical Sites: Department of Ophthalmology, Faculty of Medicine in Hradec Kralove, Charles University in Prague and University Hospital in Hradec Kralove, Czech Republic: Jan Studnicka MD., Ph.D., Jaroslava Dusova MD., Ivana Cermanova, Gabriela Blazkova; Department of Ophthalmology, First Faculty of Medicine, Charles University in Prague and General University Hospital in Prague, Czech Republic: Zora Dubska, MD., Ph.D., Bohdan Kousal, MD.; Department of Ophthalmology, University Hospital, Olomouc, Czech Republic: Assoc. prof. Jiri Rehak, MD., Ph.D., FEBO, Oldrich Chrapek, MD., Ph.D., Zuzana Pracharova, MD., Martin Sin, MD.; Department of Ophthalmology, First Faculty of Medicine, Charles University in Prague and Central Military Hospital in Prague, Czech Republic: Jan Ernest, MD., Ph.D.; Department of Ophthalmology, University Hospital, Brno, Czech Republic: Petr Kolar, MD., Ph.D., Daniela Vyslouzilova, MD., Veronika Matuskova, MD.; Department of Ophthalmology, Masaryk Hospital, Usti nad Labem, Czech Republic: Martin Hovorka, MD., Martina Zavorkova, MD.; Department of Ophthalmology, University Hospital, Ostrava, Czech Republic: Jan Nemcansky, MD., Pavel Smehlik, MD.; Department of Ophthalmology, Faculty of Medicine in Plzen, Charles University in Prague and University Hospital in Plzen, Czech Republic: Dagmar Frdlikova, MD., Hana Fidranska, MD., Tomas Nathansky, 
MD.; Department of Ophthalmology, Teaching Hospital Kralovské Vinohrady, Prague, Czech Republic: Miroslav Veith, MD., Stanislava Pokorna, MD.

\section{REFERENCES}

1. Seddon JM, Chen CA. The epidemiology of age-related macular degeneration. Int Ophthalmol Clin 2004;44:17-39.

2. Ferris FL III, Fine SL, Hyman L. Age-related macular degeneration and blindness due to neovascular maculopathy. Arch Ophthalmo 1984;102:1640-2.

3. Wong T, Chakravarthy U, Klein R, Mitchell P, Zlateva G, Buggage R Fahrbach K, Probst $C$, Sledge I. The natural history and prognosis of neovascular age-related macular degeneration. Ophthalmology 2008;115: 116-26.

4. Ng EW, Shima DT, Calias P, Cunningham ET Jr, Guyer DR, Adamis AP. Pegaptanib sodium, a targeted anti-VEGF aptamer for ocular vascular disease. Nat Rev Drug Discov 2006;5:123-32.

5. Lowe J, Araujo J, Yang J, Reich M, Oldendorp A, Shin V, Quarmby V, Lowman H, Lien S, Gaudreault J, Maia M. Ranibizumab inhibits multiple forms of biologically active vascular endothelial growth factor in vitro and in vivo. Exp Eye Res 2007;85:425-30.

6. Treatment of age-related macular degeneration with photodynamic therapy (TAP) Study Group. Photodynamic therapy of subfoveal choroidal neovascularization in age-related macular degeneration with verteporfin: one-year results of 2 randomized clinical trials - TAP report. Arch Ophthalmol 1999;117:1329-45.

7. Holz FG, Amoaku W, Donate J, Guymer RH, Kellner U, Schlingemann RO, Weichselberger A, Staurenghi G. Safety and efficacy of a flexible dosing regimen of ranibizumab in neovascular age-related macular degeneration: the SUSTAIN study. Ophthalmology 2011;118:663-71.

8. Fung $A E$, Lalwani $G A$, Rosenfeld PJ, Dubovy SR, Michels S, Feuer WJ, Puliafito CA, Davis JL, Flynn HW Jr, Esquiabro M. An optical coherence tomography-guided, variable dosing regimen with intravitreal ranibizumab (Lucentis) for neovascular age-related macular degeneration. Am J Ophthalmol 2007; 143:566-83.

9. Gragoudas ES, Adamis AP, Cunningham ET Jr, Feinsod M, Guyer DR. Pegaptanib sodium for neovascular age-related macular degeneration. N Engl J Med 2004;351:2805-16.

10. Quiram PA, Hassan TS, Williams GA. Treatment of naïve lesions in neovascular age-related macular degeneration with pegaptanib sodium. Retina 2007; 27:851-6. 